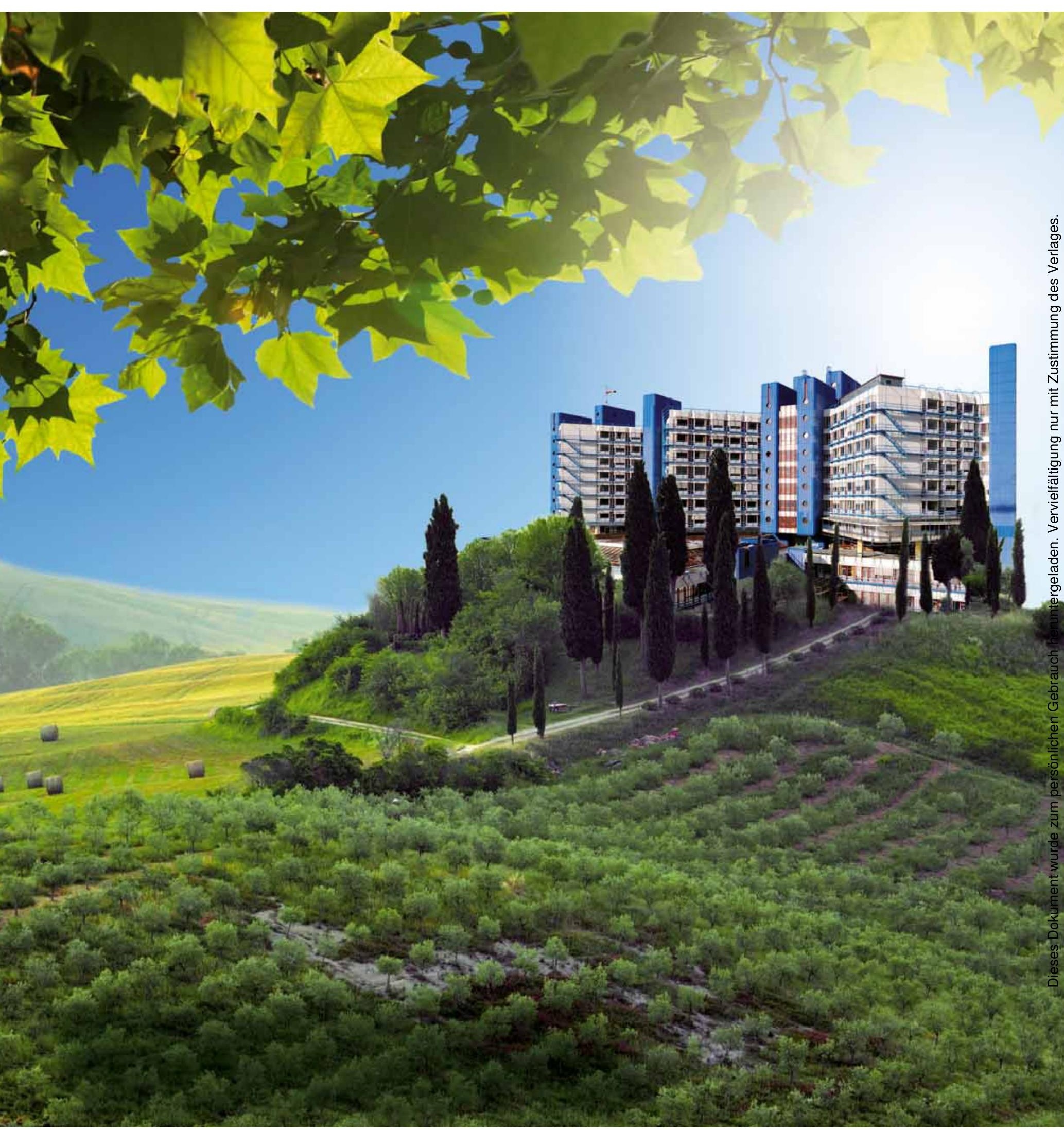

„Die neue Betriebsform war ein Aufbruch, und dieser Aufbruch hat uns gerettet." 


\title{
Renaissance der Stiftung
}

\author{
Ein klassisches Trägermodell in neuer Form kann \\ kommunale Krankenhäuser aus den Klauen der Politik \\ befreien, handlungsfähiger und nachhaltig machen. \\ Bamberg hat es vorgemacht. Hessen arbeitet daran.
}

D ie Nerven lagen blank, in Bamberg, im Jahr 2004. In Zeitungsberichten über Bayerns fünftgrößtes kommunales Klinikum sah man Fotos von leeren Betten. Vier Stationen waren bereits geschlossen, das OPProgramm war drastisch reduziert, die Angst vor betriebsbedingten Kündigungen ging um - und die Angst, verkauft zu werden. Mit der Einführung der DRG-Fallpauschalen war das Haus plötzlich nicht mehr ausgelastet. Die Patienten blieben kürzer, wenn sie überhaupt kamen und nicht im ambulanten Sektor hängenblieben. Zugleich konnte niemand mehr die Augen davor verschließen, dass das 1984 eröffnete Klinikum in die Jahre gekommen war. Dreibettzimmer ohne Nasszelle; klassisches Stationsbad; Warteschlangen vor den Aufzügen; und ein Jahresverlust von vier Millionen Euro: höchste Eisenbahn, dass jemand handelte - und zwar entschlossen.

\section{Historische Erfahrung}

Not macht erfinderisch, sagt ein Sprichwort. In einer Mischung aus purer Verzweiflung, der Flucht nach vorne und der vielleicht etwas späten wie banalen Einsicht, dass ein Krankenhaus-Management nur handeln kann, wenn man es auch lässt, rangen die Lokalpolitiker um die richtige Lösung. Zur Debatte standen die Umwandlung des damaligen städtischen Eigenbetriebs in eine
GmbH. Und eine Veräußerung. Am Ende entschlossen sie sich, das Klinikum nicht zu privatisieren und dennoch loszulassen, indem sie es der von ihnen selbst gegründeten „Sozialstiftung Bamberg" (SSB) überantworteten.

Das Stiftungsmodell als neue Trägerform für ein modernes Krankenhaus: Das war eine für Deutschland ungewöhnliche Lösung; aber in Bamberg wurde sie als Option erkannt, weil man mit dem Modell an sich nicht fremdelte. Die wirtschaftsstarke Universitäts- und Erzbistumsstadt besitzt eine historische Erfahrung und Tradition mit Stiftungen. Teil der neuen Stiftung sind drei weitere, zuvor existierende, die Seniorenheime betrieben, und Deutschlands zweitälteste Nervenklinik. Auch wenn sich die Stadträte damals nicht sicher sein konnten, ob am Ende alles gut geht und aus der Not eine Tugend machten: Sie legten mit ihrem Votum für die alternative Unternehmensform den Grundstein für die Rettung des Klinikums und dessen nachhaltige Existenzfähigkeit. Erklärtes Ziel der neuen Rechtsform: eine moderne Unternehmensstruktur schaffen und die Kliniken und Heime fit machen für den Wettbewerb. „Die neue Betriebsform war ein Aufbruch, und dieser Aufbruch hat uns gerettet", sagt Xaver Frauenknecht, Vorstandsvorsitzender der Sozialstiftung Bamberg. 


\section{Jetzt buchen: Ihre Werbung in kma}

in der kma krankenhaustechnik zum Jubiläum der FKT

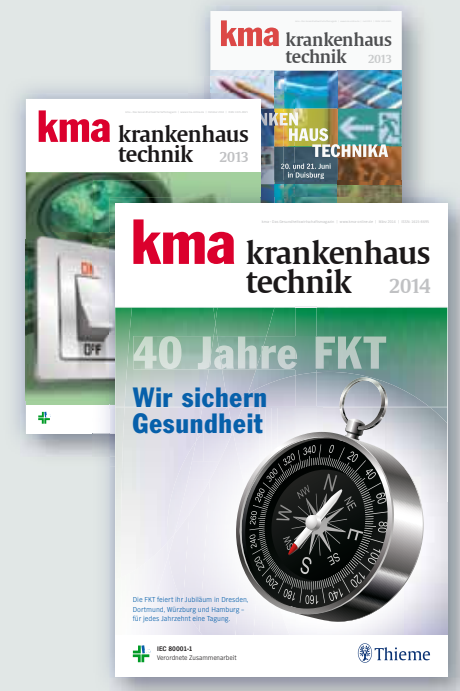

Anzeigenschluß: 8. April 2014

Wir bieten zweimal im Jahr praxisorientierte, topaktuelle Informationen zum technischen Gebäudemanagement im Gesundheitswesen.

Wir sind Ihr direkter Draht zu den Entscheidern im Krankenhaus buchen Sie jetzt Ihren Medienauftritt!

Setzen Sie sich direkt mit Ihrem Ansprechpartner in Verbindung: Imke Ridder, Anzeigenverkauf kma-fkt@imke-ridder.de | Telefon: 08194/20 7735

\section{kma}
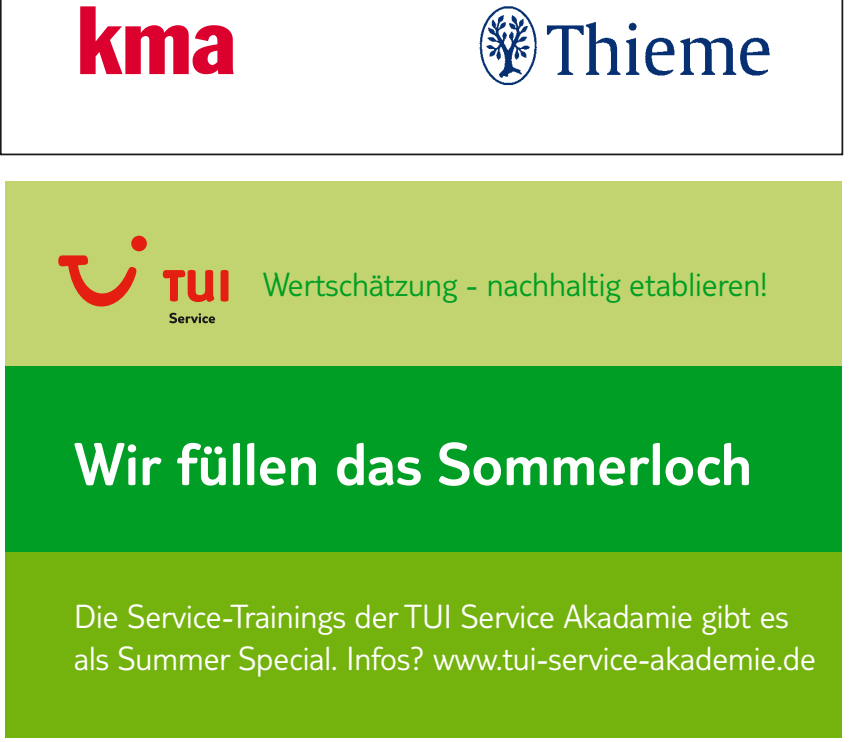

Xaver Frauenknecht: „Die Stiftung als Unternehmensform ist eine gute Alternative, wenn es darum geht, nachhaltig zu wirtschaften und die Krankenversorgung als Daseinsvorsorge sicherzustellen."

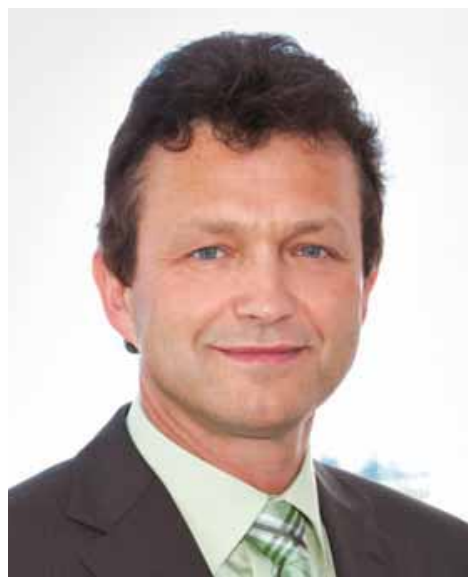

„Wir sind damals unterkapitalisiert hineingegangen, mit defizitären Betrieben. Jetzt, nach zehn Jahren, befinden wir uns auf einem soliden Kurs. Im achten Jahr hintereinander haben wir ein positives Betriebsergebnis erzielt.“ Eine Änderung der Unternehmensform allein löst die Probleme nicht automatisch. Aber Frauenknecht sagt: „Es hängt mit der Stiftung zusammen, ganz eindeutig.“ Jede Stiftung dient einem definierten „Zweck“, in unserem Fall: der Versorgung von Kranken und Alten, verstanden als Daseinsvorsorge. „Den - und nur den hat sie zu erfüllen“, sagt Frauenknecht, dessen Aufgabe darin besteht, das Reinvermögen nachhaltig zu bewirtschaften und zu erhalten, „und deshalb müssen wir Gewinn machen, natürlich.“ Der Manager, der im Lauf seiner Karriere auch andere kommunale und private Klinikträger von innen kennengelernt hat, sagt über den Charme des Stiftung: „Ihr gesamter Einsatz gilt dem Patienten, den Bewohnern, den Mitarbeitern. Wir brauchen keine übertriebene Rendite. Es gibt keine Gewinnentnahme wie in einer Aktiengesellschaft. Weil wir alle Erlöse zweckbezogen weiterverwenden müssen, können wir auch jeden Cent, den wir erwirtschaften, in dieses Unternehmen reinvestieren. Und anders als in einer GmbH gibt es auch keine direkte Gesellschafterweisung. Das ist der entscheidende Punkt.“

\section{"Eine Stiftung gehört sich selbst“}

Kliniksprecherin Brigitte Dippold sagt pointiert: „Eine Stiftung gehört niemandem. Eine Stiftung gehört sich selbst.“ Und es gibt nur zwei Organe: den Vorstand eben und den Stiftungsrat. Mit dem Oberbürgermeister und sieben Stadträten stellt die Lokalpolitik zwar auch hier weiterhin die Mehrheit. Aber erstens ist das Gremium universeller besetzt (auch mit Vertretern von Personal, regionaler Wirtschaft und dem Bezirkstag). Und zweitens sind dessen Kompetenzen so klar definiert wie begrenzt. Klinikchef Frauenknecht sagt: „Der Stiftungsrat gibt die Unternehmensstrategie vor. Mit der operativen Umsetzung hat er nichts zu tun.“

Die Lasten aus Zins und Tilgung für seit Jahren aufgelaufene Schulden, fehlende Mittel für notwendige Investitionen 


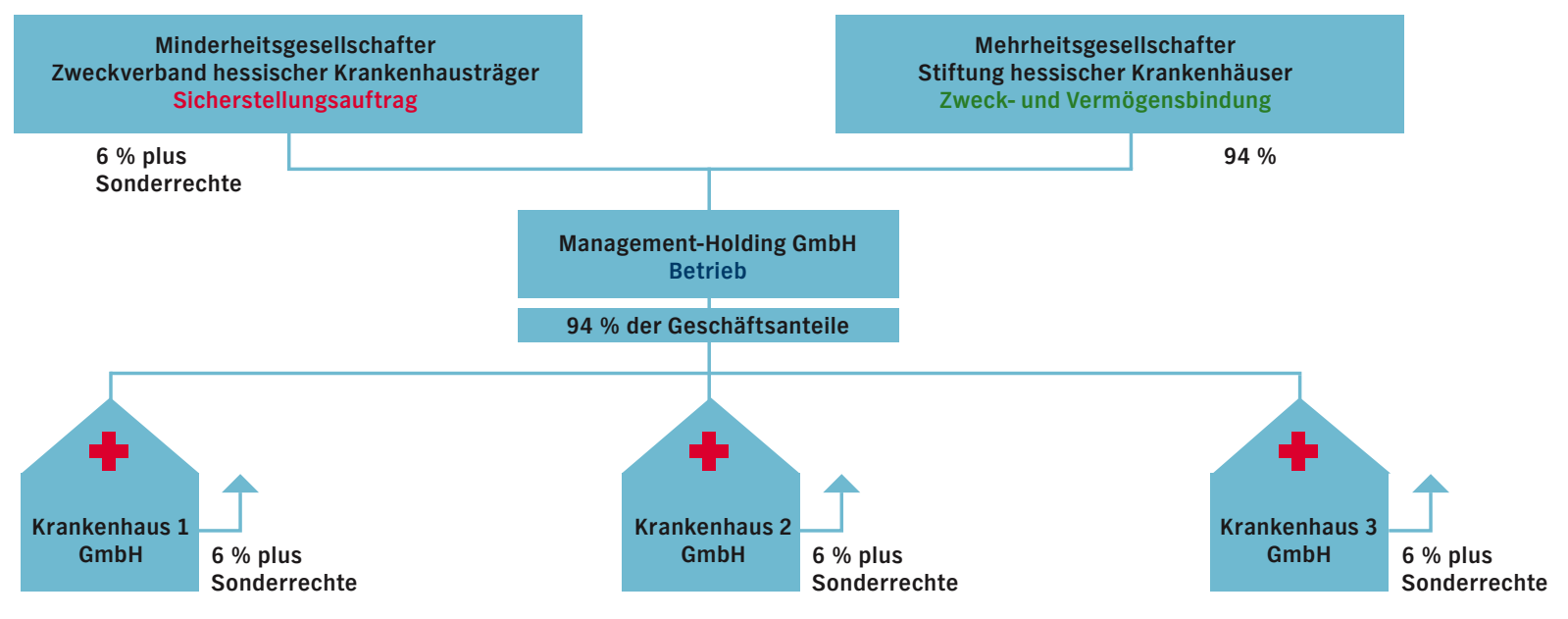

Quelle: Luther Rechtsanwaltsgesellschaft

Ausweg aus dem „roten Bereich“: Nach dem von der Anwaltssozietät Luther in Frankfurt vorgelegten Konzept könnte der erste Schritt zur landesweiten Krankenhausstiftung darin bestehen, dass eine Kommune die Geschäftsanteile an ihrer Krankenhausgesellschaft mehrheitlich in die Management-Holding GmbH einbringt, beispielsweise zu 94 Prozent. Einen „Zwerganteil“ von sechs Prozent behält sie, denn dieser sichert ihr gewisse Sonderrechte - etwas das Veto-Recht zu verhindern, dass die Holding ihre Anteile an der Krankenhausgesellschaft an einen Dritten veräußert. Die Aufgabe der Holding ist es, den Verbund zu steuern und die wesentlichen unternehmenspolitischen Entscheidungen zu treffen. Einzelentscheidungen des klinischen Tagesgeschäfts sollen weiterhin in den einzelnen Krankenhäusern getroffen werden. Mehrheitsgesellschafterin der Holding ist die Stiftung Hessischer Krankenhäuser. Die garantiert, dass das eingebrachte Vermögen ausschließlich für die bestmögliche Umsetzung des Stiftungszwecks verwendet wird - des Sicherstellungsauftrags für die Gesundheitsversorgung der Bevölkerung. Minderheitsgesellschafter der Holding ist ein Zweckverband, zu dem sich die beteiligten Kommunen zusammenschließen und auf den sie ihren Sicherstellungsauftrag übertragen.

und sachfremde politische Interventionen ins operative Geschäft: „Dies sind die großen Schwachstellen der Krankenhäuser in öffentlicher Trägerschaft“, sagt Thomas Köhler, Spezialist für das Gesundheitswesen im Frankfurter Büro der Luther Rechtsanwaltsgesellschaft. Köhler ist federführend verantwortlich für ein im Auftrag des hessischen Sozialministeriums erarbeitetes Zukunftskonzept für den Erhalt kommunaler Kliniken, das derzeit dort umgesetzt wird. Hessen gehört zu den Gegenden, in denen der Leidens- und Handlungsdruck bei den kommunalen Krankenhäusern am größten ist. Auf einer Deutschlandkarte im Krankenhaus Rating Report 2013 des RWI Essen gehört Hessen zu den Regionen, die dunkelrot eingefärbt sind. Hier liegt der Anteil der öffentlich-rechtlichen Krankenhäuser, die in Gefahr sind, in der Klasse „35 Prozent oder mehr" und erreicht damit Spitzenwerte. Die Ausfallwahrscheinlichkeit für ein öffentliches Haus ist dem Report zufolge in Hessen um fast 60 Prozent höher als im Bundesdurchschnitt. Diese desolate Situation hat dazu geführt, dass Köhler in seinem Überlebenskonzept vorschlug, einen völlig neuen Weg zu gehen: mit einem Krankenhausverbund, an dessen Spitze eine Stiftung steht. Bei dieser Konstruktion geht Hessen in seiner Not noch einen Schritt weiter als Bamberg: Im Kontrollgremium der Stiftung, das die strategischen Entscheidungen fällt, sollen „im Gesundheitswesen bewanderte Fachleute die Mehrheit haben“, sagt Jochen Metzner, Leiter des Referats Krankenhausversorgung im hessischen Sozialministerium. „Die Kommunalpolitik ist vertreten und hat Rede- und Stimmrecht aber sie wird in der Minderheit sein.“

\section{Die Praxis steht noch aus}

Was in der Theorie gut klingt, muss sich in der Praxis erst noch beweisen. Gerhard Sontheimer, Vorstandschef der "Gesundheit Nord Holding“ (GNH) und damit des größten regionalen Verbunds in Hessen, lehnte einen Beitritt seines Unternehmens ab. Die GNH im Raum Kassel gehört zu den wenigen großen kommunalen Krankenhäusern in Hessen, die schwarze Zahlen schreiben. Die Geschäftsführung des GPR-Klinikums Rüsselsheim begrüßte das Konzept als hilfreichen Anstoß, über die Zukunft der kommunalen Häuser nachzudenken, lehnte einen Beitritt allerdings ebenfalls ab. Nach Auskunft aus dem Wiesbadener Sozialministerium bekundeten bisher 13 der 26 öffentlichrechtlichen Krankenhausunternehmen ernsthaftes Interesse an dem neuen Modell und nahmen an einer Unternehmensbewertung durch PricewaterhouseCoopers teil, die schon einmal eine Entscheidungsbasis für einen potenziellen Beitritt darstellen kann. 


\section{Krankenhausfinanzierung durch Frankenwein}

Eine Stiftung ist eine Einrichtung, die mithilfe eines Vermögens einen vom Stifter festgelegten Zweck verfolgt. Stiftungen können in verschiedenen Rechtsformen und zu praktisch jedem legalen Zweck errichtet werden. Stiftungen öffentlichen Rechts stellen neben den Körperschaften und den Anstalten einen Organisationstyp öffentlich-rechtlicher juristischer Personen dar. Sie werden vom Staat durch Gesetz oder Rechtsverordnung errichtet und besitzen oft lediglich ein Sachvermögen - Immobilien etwa. Die meisten Stiftungen werden in privatrechtlicher Form errichtet, 95 Prozent davon dienen gemeinnützigen Zwecken - nicht wenige seit Jahrhunderten. Nach Auskunft des Bundesverbands Deutscher Stiftungen existieren derzeit 20.150 rechtsfähige Stiftungen bürgerlichen Rechts mit einem Vermögen von rund 70 Milliarden Euro. Allein im vergangenen Jahr kamen 638 neue hinzu. 207 Stiftungen dieses Typs betreiben Krankenhäuser. Gemessen am Vermögen ist die Robert-Bosch-Stiftung die größte (5 Milliarden Euro), gemessen an den jährlichen Gesamtausgaben die Volkswagen-Stiftung (150 Millionen Euro). Mit 75 Stiftungen pro 100.000 Einwohner verfügt Hamburg über die mit Abstand größte Stiftungsdichte aller Bundesländer. Stiftungshauptstadt ist Würzburg mit einer Dichte von 90 pro 100.000 Einwohner. Die berühmteste Institution ist hier die

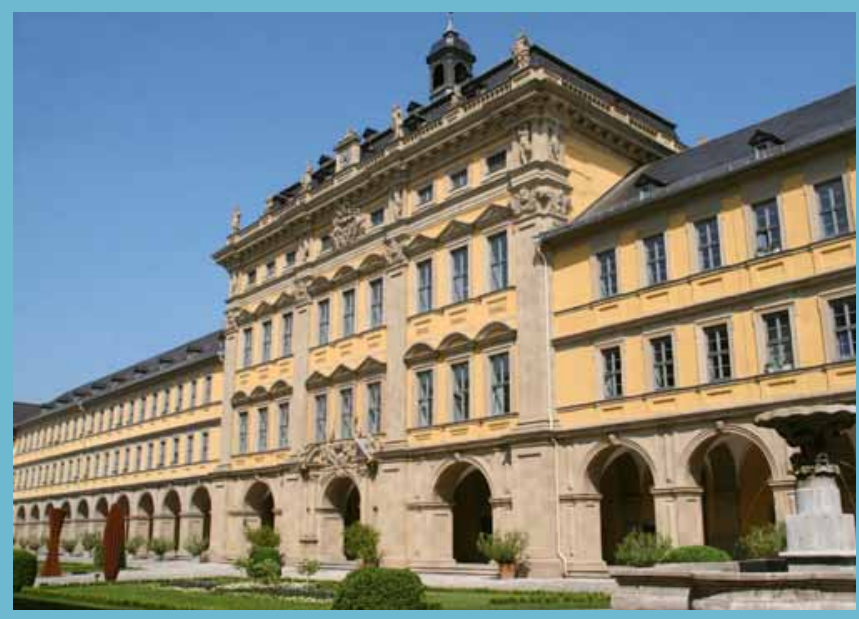

Fürstlich: Die Stiftung Juliusspital in Würzburg kümmert sich seit 438 Jahren um Kranke, Alte und Bedürftige. In den Kellergewölben unter dem Krankenhaus lagert in Holzfässern der Wein, der seit 1576 einen respektablen Beitrag zur Finanzierung von sozialen Aufgaben leistet.

Stiftung Juliusspital, die 1576 vom damaligen Fürstbischof Julius Echter gegründet wurde. Kernstück ist das gleichnamige Krankenhaus der Schwerpunktversorgung in der Innenstadt mit 342 Betten. Die Stiftung finanziert sich aus den Erträgen von 4.500 Hektar Grundbesitz - Wäldern, Feldern und Weinbergen. Das Juliusspital zählt zu Deutschlands Spitzenweingütern und gilt als zweitgrößtes Weingut bundesweit.
Um Häuser zur Teilnahme zu animieren, hatte sich das Land Hessen von vornherein bereiterklärt, die Kosten für die Expertise zu übernehmen.

„Der Name ,Stiftung' ist noch nicht die Lösung “: Zu gesunder Skepsis fordert Claus Bartels die Beteiligten auf, dessen Consultingfirma Med Advisors im Auftrag des Landesrechnungshofs Hessen sieben kommunale Kliniken unter die Lupe genommen hat. „Das primär Entscheidende ist, dass solche Modelle vernünftig ausgestaltet sind. Erfolgt das nicht, ist es hier genauso schwierig zu agieren wie in einer Anstalt öffentlichen Rechts oder einer GmbH." In Hessen sind aus seiner Sicht „zentrale Dinge noch nicht transparent gemacht oder nicht geklärt", die über Erfolg oder Misserfolg dieses als Stiftung angedachten Verbunds aber mit entscheiden: die wirtschaftliche Situation der Einzelhäuser, der

\section{"Der Name ,Stiftung، ist noch nicht die Lösung.} Sie muss auch vernünftig ausgestaltet sein."

Claus Bartels, Med Advisors

Investitionsstau für das Gesamtgebilde, der Umgang mit überschüssigem Personal nach einer Zusammenlegung redundanter Unternehmensbereiche, vor allem in Tertiärbereichen, oder eine geografische Neusortierung und Straffung des medizinischen Sortiments durch den Landesgesetzgeber im Krankenhausbedarfsplan.

\section{Alternative zur Privatisierung}

„Ein überregionales kommunales Verbundmodell, das die besonderen Werte öffentlicher Krankenhäuser bewahrt, diesen Werten auf allen Entscheidungsebenen verpflichtet ist und die Kommunen gleichzeitig dauerhaft von ihrem Sicherstellungsauftrag entlastet, ist ein Pilotprojekt“, sagt
Rechtsanwalt Köhler. Der Gesundheitsexperte rechnet damit, dass das Modell über die Landesgrenzen hinaus Schule machen könnte, weil es für Kommunen eine echte Alternative zur Privatisierung sei. Auch Claus Bartels sympathisiert unbeschadet seiner kritischen Bemerkungen zu den Hausaufgaben, die in Hessen noch zu machen sind, mit dem Stiftungsmodell. Er selbst räumte seinen Posten als Vorstandschef des Uniklinikums Greifswald nach dessen Eingliederung in die neue Universitätsmedizin. Bartels ging unter Protest: Er sah die akute Gefahr, dass ihm über die Aufsichtsgremien Fachfremde ins Alltagsgeschäft hineinregieren könnten. Deshalb sagt 


\section{Wirksame Führung lernen}

113 Produktivität und Wirtschaftictikeit im DRG-System

viter-Bund. dem Deutschen Roten Kreux oder kurztristig frel. So konnten u.a. Hitsseinsales.

nitrea und Indien personeth westis hatus ist die Id medizinisch verantwortlich. Die Ourchlo Kint mocthigen Arbeit vor Ort wird foldsejplinen der Gemeinsam mit anderen fasen währead eines ird eine grobe Anzalit Patic

Weiterbildung. und Weiterbila Tätigkei

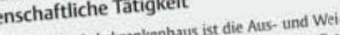
ademisches Letrikar. Prakrikanten aus dem Rer dung vor Mitarbeitent, Mrenten ein weiteres wichtil dienst sowie Medizins im Stinne der Persobalanwer. Mehrmals jathrich veranstaltet die Kinik jwe Gige Seminare zum Thema ultraschallacesmt 6 Mitalanasthesie und Kinderanasthesic. Is er vermitch praw aus dem thementereich

\section{Cretrien}

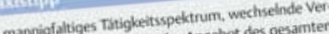

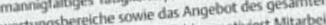
the is

1.2 Leistungs- und Costenmanagement

政

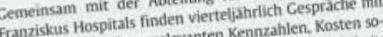
Nalyse der abteilungstelevanten Kennzahien, Kostenale ic Leistungsdaten statt. Makgeblichere Gesphan vorher-

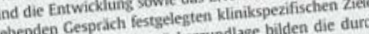
Reurteilungs- und Cesprachsgrungezeigten Kennzahles das Controlling in - Abiekcorium effolgt die Definition

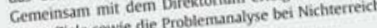
never Zueie sam

ings, sich nicht allein sur die Steifer faltzati $z u$ fokussieren, sonderm den Eriosoteil pro Fall zu maximieren und den dafur nobtigert Narwand zu minimesstandards
11.3 Produktivität und Wirtchaftlichkeit im DRG-System Die Produktivitat und kostenefliziente Leistungsetbriatung sicher dir Wisc Wirtschattictikeir bedeuten mitchW thinit und Ausbieiben von thvesti.

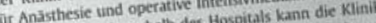
is Dienstlester innerthatb desiren und ist sonit darate eine eigenen ratienten ahyundenen operativen facher angewiesen, dass de und sozial-kompetent gute teiste.

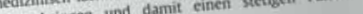
gen ertoringent unnen.

Withigste Aufgabe der Klinik fir Anasthesie und opera-

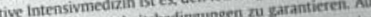
niken optimale Arbersbistandards, die eine maximal gezeichnere Vess an Operationen pro Operationsual sind effizizente post

Ine kosteneffiziente intersivivedizinische Patientenversorgung kann nur durchie enger Kooperation mit densprectenosen station seicht werden. Wictitig und finan.

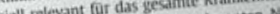

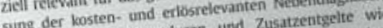
komplizierenden Prozeduren und zasspachparaten. InTransfusionen und Gabe von Gerctionen. Sepsis, Blutume raoperative Dlagnosen w Storungen des Wasser-und Bercerintungsstores soltren ebenfalls lackentos eriast une trolychaushiles ste Maknatimen und Therapien en

pie Erfassung der relevanten Nebendiagnosen und Konn-

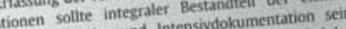
nischen Anastitiesie.

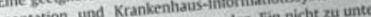

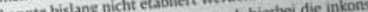
charzender Schwachpunkt ist ankth hive durchyeführten tente Dokumentation der periopetakre distren ProzedLeistungert, in Verbind

ren und Diagnosen.

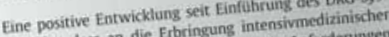
ems ist, dass an die Erbringung intensin Anforderungen und schimerztherapentisther Lnd Dokumentation gerchen.

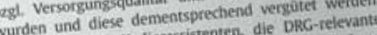

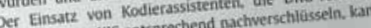

Management-Handbuch für Chefärzte

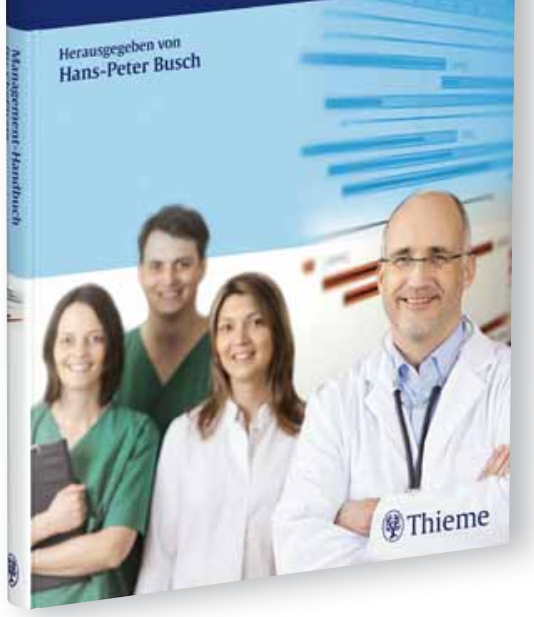

\section{Pflichtlektüre für Chefärzte und leitende Oberärzte}

- Fachwissen zu Budgetverhandlung, flexibler Plankostenrechnung, Kosten-/Erlösrechnung, u.v.m.

- Werkzeugkasten für das strategische Management: Prozessoptimierung, Behandlungspfade, Benchmarking

- Praxistipps für das operative Management und systematische Fallstudien

Mit Geleitwort von Prof. Dr. Fredmund Malik, mehrfach ausgezeichnetem Bestsellerautor von Management-Handbüchern

Management-Handbuch für Chefärzte

Busch

2012. 232 S., 110 Abb., geb.

385 S., 745 Abb., kart.

ISBN 9783131622617

$149,99 €[D]$

$154,30 €[A] / 210,-C H F$

\section{Versandkostenfreie Lieferung innerhalb Deutschlands!}




\section{Sehr bewusst gestrickt}

Früher gab es keine Stiftungsaufsicht und keinen Paragrafendschungel. Historische Sozialstiftungen begannen einfach; moderne dagegen müssen vorausschauend definiert werden. Ein Gespräch mit Rechtsanwältin Gabriele Ritter, BDO Köln.

\section{Interview mit Gabriele Ritter}

Was ist der Unterschied zwischen der caritativen Stiftung von einst und der Stiftung als Unternehmensform von hente?

Früher gab es einen altruistischen Stifter, der wollte etwas Gutes tun, gab Geld oder Vermögen, und dann startete man einfach das Projekt. Im Vordergrund stand eine hohe soziale Verantwortung, über wirtschaftliche Komponenten machte sich niemand Gedanken, gesetzliche Reglements gab es nicht. Die Krankenhäuser von heute aber sind Wirtschaftsunternehmen, die am Markt bestehen müssen. Wenn ich heute eine Krankenhausstiftung gründe, ist sie ein Rechtssubjekt. Das waren Stiftungen früher eher nicht. Ich muss mir deshalb sehr klare Gedanken machen und die Zweckausrichtung gut und vorausschauend definieren. Die Stiftungen von heute sind viel bewusstere Konstruktionen, die sich in einem Regelungsgeflecht und gegenüber der Stiftungsaufsicht dauerhaft behaupten können müssen.

Was ist interessant an der Stiftung als Rechtsform für den modernen Krankenhausbetrieb?

Die Stiftung ist eine Gesellschaftsform, die den modernen Anforderungen an ein effizientes und straffes Krankenhausmanagement bereits durch ihre innere Struktur entgegenkommt. Weil die Stiftung über keine Gesellschafter verfügt, ist die zentrale
Entscheidungsperson der Vorstand. Das bedeutet: höchstmögliche Entscheidungsfreiheit der Leitungsebene. Mit der Stiftung als Rechtsform können Kommunen außerdem ihren Auftrag der Gesundheitsvor- und -fürsorge

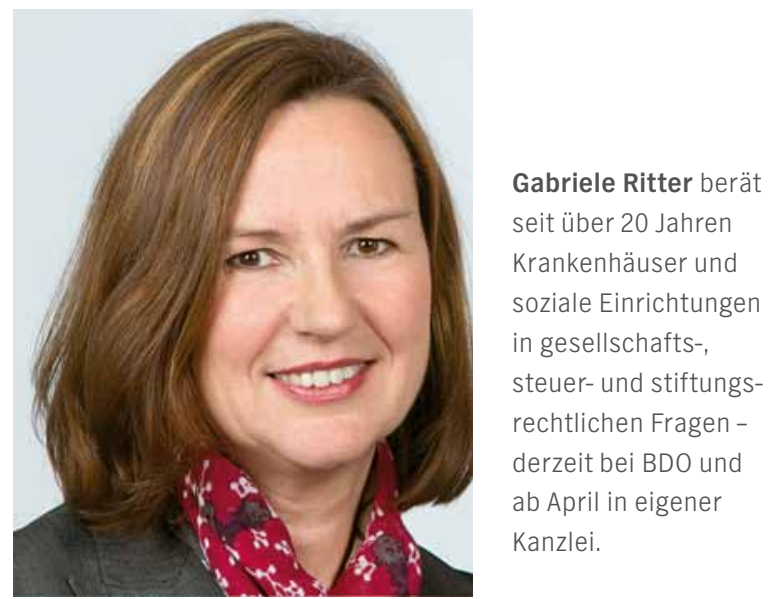
auf Dauer zementieren. Und da stiftungsrechtlich nicht ohne weiteres verkauft werden kann, gibt es auf dem freien Markt auch keine Begehrlichkeiten. Und: Die Stiftung ist eine Rechtsform, die bei der Bevölkerung gut ankommt, weil sie Seriosität verspricht.

Gibt es auch Umstände oder Krankenhäuser, für die sich die Stiftung weniger empfieblt?

Wenn ein Träger sich nicht definitiv sicher ist, ob er ein Krankenhaus nicht einmal verkaufen will, oder wenn daran gedacht wird, sich auf mittlere Sicht vielleicht einem Verbund anzuschließen, dann muss man sehr gut analysieren, ob die Stiftung die geeignete Rechtsform ist.

\section{Welche Dinge sind zu beachten?}

Ein Problem ist ja, dass - bei kommunalen Stiftungen - die Kommune im Prinzip eine Institution gründet, an der sie nachher nicht mehr beteiligt ist. Deshalb muss zunächst geklärt werden, ob die Stiftungslösung mit dem Kommunalrecht, das ja von Bundesland zu Bundesland variieren kann, in Einklang steht. Und eine weitere wichtige Frage ist: Führe ich das Krankenhaus oder gegebenenfalls meine Beteiligung daran dem Grundstockvermögen der Stiftung zu oder nicht? Eine Stiftung braucht ja ein solches Vermögen. Wenn ja, muss ich es durch einen Wirtschaftsprüfer bewerten lassen.

Manche bezeichnen die Stiftung als ein Zukunftsmodell, insbesondere für kommunale Krankenbäuser. Was ist Ibre Position dazu?

Die Stiftung ist eine interessante Rechtsform, und sie ist wieder en vogue. In den vergangenen Jahren hat sich bei den Stiftungen eine gewisse Eigendynamik entwickelt, auch als Rechtsform für Wirtschaftsunternehmen insgesamt. Stiftungen an sich unterliegen keinen Veröffentlichungspflichten, und dies gilt auch für Krankenhäuser. Wenn wir Beratungsgespräche führen über Strukturvorhaben bei Krankenhäusern und Wohlfahrtseinrichtungen, wird die Stiftung als Option regelmäßig thematisiert. Interview: Adalbert Zehnder 
Fortsetzung von Seite 36 er heute: „Für uns als Klinikchefs ist das Interessante am sinnvoll gestalteten Stiftungsmodell: Wir haben hier im Regelfall den höchsten Freiheitsgrad. Dieses Modell kann uns das Leben deutlich leichter machen.“

Als lebenden Beweis für Bartels' Einschätzung kann man Xaver Frauenknecht anführen. Mit der Gründung der Sozialstiftung Bamberg wurde er als deren Vorstandsvorsitzender berufen. Heute, zehn Jahre danach, bekleidet er das Amt noch immer. Eine Kontinuität, die sehr ungewöhnlich für die deutsche Krankenhauslandschaft ist, geht es in deren Führungsetagen doch häufig zu wie im Taubenschlag. Die in Bamberg institutionalisierte Nachhaltigkeit hat in Frauenknechts Augen entscheidend dazu beigetragen, dass aus dem Übernahmekandidaten von 2004 ein regionaler Gesundheitskonzern geworden ist. Allein das Klinikum Bamberg wurde im Rahmen eines Investitions- und Instandhaltungsprogramms von bisher 100 Millionen Euro aufgemöbelt. Es herrscht eher Hotel- als Krankenhausatmosphäre. Es gibt doppelt so viele Kliniken wie einst und alle wichtigen Fachdisziplinen außer Herzchirurgie und Transplantationsmedizin. Dazu Medizinische Versorgungszentren (MVZ) und Wahlleistungsstationen. Und statt acht Aufzügen - in Bettentürmen immer auch Lebensadern - gibt es zwölf. Mitfinanziert haben das Mammutprojekt auch die Mitarbeiter durch den deutschlandweit ersten Zukunftssicherungstarifvertrag zwischen einem Klinikum und der Gewerkschaft Verdi von 2006. Das Geld aus dem teilweisen Lohnverzicht bis 2009 haben sie inzwischen zurückbekommen. „Die
Väter und Mütter der Stadt haben sich damals ein Stück weit entmachtet", sagt Stiftungssprecherin Dippold im Rückblick. „Sie taten dem Klinikum einen Gefallen und sich selbst - auch wenn sie heute nicht mehr mitreden dürfen, wo und wie viele Blumenkübel wir vors Haus stellen."

Adalbert Zehnder

\section{Mehr Infos}

1

www.sozialstiftung-bamberg.de Sozialstiftung Bamberg

http://to.kma-online.de/zn57 Hessisches Sozialministerium Konzept für den Erhalt öffentlicher Klinikträgerschaften

www.stiftungen.org Bundesverband Deutscher Stiftungen

Die conhlT verbindet aktuelle Marktlösungen und zukunftsgerichtete Innovationstrends - auch im europäischen Kontext - mit einem umfangreichen Networking- und Weiterbildungsangebot. Für mich ein einzigartiger Fundus an Möglichkeiten. Ressortleiter IT / TK, Vivantes Netzwerk für Gesundheit GmbH Arbeitskreis Informationstechnologie der Arbeitsgemeinschaft kommunaler Großkrankenhäuser

GOLD-Partner

AGFA

Health Care

MelERHOFER
ID Information und

Dokumentation im
Gesundheitswesen

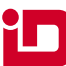

medatixx

SIEMENS R... $\begin{gathered}\text { healthcare } \\ \text { solutions }\end{gathered}$
Nur bis zum 31. März 2014!

Kostenloses Messeticket auf www.conhit.de
Organisation

IIIIII Messe Berlin 